\title{
Dahuang Zhechong Pill Combined with Doxorubicin Induces Cell Death through Regulating Energy Metabolism in Human Hepatocellular Carcinoma Cells
}

\author{
Li Wu, ${ }^{1,2}$ Jiayu Zhao, ${ }^{1,2}$ Hao Cai, ${ }^{1,2}$ Jiaqi Wang, ${ }^{1,3}$ Zhipeng Chen, ${ }^{1,2}$ \\ Weidong Li, ${ }^{1,2}$ and Xiao Liu ${ }^{1,2}$ \\ ${ }^{1}$ Department of Pharmacology, School of Pharmacy, Nanjing University of Chinese Medicine, Nanjing, Jiangsu, China \\ ${ }^{2}$ Engineering Center of State Ministry of Education for Standardization of Chinese Medicine Processing, \\ School of Pharmacy, Nanjing University of Chinese Medicine, Nanjing, Jiangsu, China \\ ${ }^{3}$ Affiliated Hospital of Integrated Traditional Chinese and Western Medicine in Jiangsu Province, \\ Nanjing University of Chinese Medicine, Nanjing, Jiangsu, China
}

Correspondence should be addressed to Hao Cai; haocai_98@126.com

Received 16 April 2017; Accepted 6 June 2017; Published 12 July 2017

Academic Editor: Yuewen Gong

Copyright (C) $2017 \mathrm{Li} \mathrm{Wu}$ et al. This is an open access article distributed under the Creative Commons Attribution License, which permits unrestricted use, distribution, and reproduction in any medium, provided the original work is properly cited.

\begin{abstract}
Many physiological activities such as cell survival, proliferation, defense, adaptation, and metabolism need to consume energy. Hepatoma cells can quickly start stress responses like multidrug resistance (MDR) requiring adenosine triphosphate (ATP) consumption after administration of chemotherapeutics. We employed CCK- 8 assay to evaluate cell viability and the flow cytometry to confirm apoptosis and necrosis. ELISA kit was used to determine intracellular levels of ATP in lysates. Western blot was employed to analyze the expressions of key enzymes involved in energy metabolism. We found that doxorubicin (DOX) potently stimulated apoptosis at a low dose and even induced necrosis at a high dose in SMMC-7721. DHZCP combined with DOX at low or middle dose enhanced the synergistic antihepatoma effect. Results indicated that Dahuang Zhechong Pill (DHZCP) inhibited the expressions of several key enzymes involved in oxidative phosphorylation and reduced intracellular ATP levels. The combination of DHZCP with DOX reversed the elevation of intracellular ATP levels, and a significantly synergistic antitumor effect was observed. DHZCP could not only strengthen the therapeutic effects of chemotherapeutic drugs but also decrease the doses of chemotherapeutic drugs and the incidences of adverse reactions, providing novel strategies for clinical treatment of liver cancer.
\end{abstract}

\section{Introduction}

In China, hepatic carcinoma, accounting for about $50 \%$ of the global incidence rate, seriously threatens people's health. It is difficult to cure HCC which are often associated with hepatic fibrosis and cirrhosis by surgery due to the insidious onset and late discovery $[1,2]$. Chemotherapy-based combination therapy is currently the major option for clinical treatment of HCC. However, there seems to be the poor effect and prognosis of HCC after chemotherapy because of the serious adverse reactions, the easy occurrence of MDR, expensive price, and so on. Seeking effective strategies for a better combination therapy against HCC is of great value for limiting the adverse reactions of chemotherapy and eradicating the $\operatorname{MDR}[3,4]$.
Traditional Chinese Medicines (TCM) have the characteristics of multicomponent, multichannel, and multitargeting, which have been widely used as an important supplement and replacement therapies of a variety of cancers including HCC. TCM may also be effective for reversal of MDR. DHZCP is one of the most commonly used TCM prescriptions in clinical adjuvant therapy for HCC. A number of clinical investigations suggest that DHZCP can significantly reduce tumor volume, prolong survival time, enhance the efficacy, and reduce the MDR, which can be more effective when combined with several chemotherapeutic drugs $[5,6]$. Nevertheless, the potential mechanism is still unclear. Our work aimed to ascertain the synergistic antitumor effects of DHZCP combined with DOX in SMMC-7721 and explore 
the potential molecular mechanisms. The experimental data from our laboratory provided the novel therapeutic insights into the clinical treatment of HCC.

\section{Materials and Methods}

2.1. Reagents and Antibodies. DHZCP (including twelve traditional Chinese medicinal herbs: Eupolyphaga Steleophaga, Rhei Radix et Rhizoma, Scutellariae Radix, Glycyrrhizae Radix et Rhizoma, Persicae Semen, Armeniacae Semen Amarum, Paeoniae Radix Alba, Rehmanniae Radix, Toxicodendri Resina, Tabanus bivittatus Matsumura, Hirudo, and Holotrichia diomphalia Bates) was purchased from Beijing TongRenTang Co., Ltd. (Lot number 15013005). DOX was purchased from Meiji Seika Kaisha Co., Ltd. (Lot number CDXB21217), and was dissolved in sterilized water for experiments. Enhanced ATP Assay Kit was purchased from Shanghai Biyuntian Biological Co., Ltd. Antibodies against complex $\mathrm{I} \sim \mathrm{V}$, hexokinase 2 (HK2), phosphofructokinase (PFKM), pyruvate kinase 2 (PKM2), and $\beta$-actin were obtained from Proteintech Group (Chicago, IL, USA).

Cell Culture. SMMC-7721 was obtained from the Cell Bank of Chinese Academy of Sciences (Shanghai, China). They were grown under standard cell culture conditions plus 10\% FBS with $100 \mathrm{U} / \mathrm{mL}$ of penicillin and $100 \mathrm{U} / \mathrm{mL}$ streptomycin, at $37^{\circ} \mathrm{C}$ in a humidified incubator with $5 \% \mathrm{CO}_{2}$.

2.2. Preparation of DHZCP-Medicated Serum. DHZCP is mixed with water to form mixed suspension, so we treat cells with DHZCP-medicated serum. Twenty-four pills and $300 \mathrm{~mL}$ ultrapure water were mixed to obtain a suspension of DHZCP. Healthy SD rats received intragastric administration of DHZCP $(240 \mathrm{mg} / 100 \mathrm{~g} /$ day, referring to clinical treatment dose) 2 times a day in seven consecutive days. After administration, rats fasted for $12 \mathrm{~h}$, and water was provided at will. After $2 \mathrm{~h}$ of last intragastric administration, the blood was taken from rats' artery under anesthesia and the serum was separated and centrifuged at $4^{\circ} \mathrm{C}, 3000 \mathrm{r} / \mathrm{min}$ for $5 \mathrm{~min}$, and the upper serum was incubated and inactivated at $56^{\circ} \mathrm{C}$ followed by filtration with $0.22 \mu \mathrm{m}$ microfiltration membrane and then preserved at $-20^{\circ} \mathrm{C}$. All procedures and experiments of this study were consented by the Animal Care and Use Committee of Nanjing University of Chinese Medicine, and its approved protocol is "Scientific Protocol (2007) Number 16 of Nanjing University of Chinese Medicine." For quality control analysis, methanol of $300 \mu \mathrm{L}$ was added to $100 \mu \mathrm{L}$ DHZCP-medicated serum and mixed for $3 \mathrm{~min}$ and then was subjected to centrifugation for $3 \mathrm{~min}$ at $12000 \mathrm{r} / \mathrm{min}$. The supernatant was detected by HPLC coupled with UVD.

2.3. Cell Viability Assay. SMMC-7721 cells were seeded in 96well cell plates, cultured in DMEM for $24 \mathrm{~h}$ to achieve $80 \%$ coverage, and then treated with the drug for 12 or $24 \mathrm{~h}$. The drug was set into eight groups: control group, DHZCP group (10\%), DOX groups $(0.5,2$, and $8 \mu \mathrm{M})$, and mixture groups [10\% DHZCP combined with DOX $(0.5,2$, and $8 \mu \mathrm{M})]$. Cell viability was measured by the method of CCK-8. Each group had six replicates. Every experiment was conducted for three times.
2.4. Flow Cytometric Analysis. SMMC-7721 cells were cultured with DHZCP-medicated serum, DOX, or their combination at concentrations for $24 \mathrm{~h}$. After being treated, they were collected and washed and suspended in PBS. Cells $\left(0.5-1.0 \times 10^{6}\right)$ were taken to centrifuge for $5 \mathrm{~min}$ at $1000 \mathrm{~g}$ and the sediments were collected. Cells were gently suspended in $195 \mu \mathrm{L} \mathrm{V}$-FITC Annexin binding solution. After adding $5 \mu \mathrm{L}$ Annexin V-FITC conjugate, we incubated cells for $10 \mathrm{~min}$ at $25^{\circ} \mathrm{C}$ avoiding light and centrifugated them $5 \mathrm{~min}$ at $1000 \mathrm{~g}$ and the sediments were collected. Cells were suspended in $195 \mu \mathrm{L}$ V-FITC Annexin binding solution again. Mix gently in the ice bath avoiding light with $10 \mu \mathrm{L}$ propidium iodide staining solution. Apoptosis and necrosis were measured with flow cytometry. Every experiment was conducted for three times.

2.5. Determination of ATP and Protein Levels. SMMC-7721 cells were administrated with DHZCP-medicated serum, DOX, or their combination at different doses for $24 \mathrm{~h}$. Endogenous expressions of ATP in lysates of treated SMMC7721 cells were detected with an ELISA kit in conjunction with the manufacturers' instructions. Every experiment was conducted for three times.

2.6. Western Blot Analysis. Cell extracts were acquired from treated SMMC-7721 with RIPA buffer plus proteinase inhibitors. Then we resolved protein by electrophoresis on SDS-polyacrylamide gels, transferred to PVDF membrane (Millipore, MA). Interested proteins were identified by specific primary antibodies and then bind to specific secondary antibodies. Every blot was conducted for three times. The expressions of interested protein bands were examined by Image J. The changes in the density of bands were expressed as fold changes compared to the control in the blot after normalization to $\beta$-actin.

2.7. Statistical Analysis. Data were exhibited as mean standard deviation (SD). The one-way analysis of variance (ANOVA) test and $t$-test were used for comparison between groups. All statistical analyses were analyzed by SPSS 15.0 with $p<0.05$.

\section{Results}

3.1. Effects of DHZCP-Medicated Serum Combined with DOX on SMMC-7721 Cell Viability. To clarify the primary components in DHZCP-medicated serum, we developed a novel and simple method, HPLC coupled with UVD, for the determination of seven bioactive compounds through different liquid chromatographic conditions. The results are as follows (Supplementary Table 1 is in Supplementary Material available online at https://doi.org/10.1155/2017/6279576). The viability of SMMC-7721 was studied with DHZCP-medicated serum at different concentrations $(2.5 \%, 5 \%$, and $10 \%)$. Results showed that the viability of SMMC-7721 was prominently diminished after treatment with 10\% DHZCP for $12 \mathrm{~h}$ and $24 \mathrm{~h}(p<0.05)$. Effects of DOX at different concentrations $(0.5,2$, and $8 \mu \mathrm{M})$ alone and its combination with $10 \%$ DHZCP on cell viability were further evaluated in the study. The results demonstrated that DOX at 2 and $8 \mu \mathrm{M}$ significantly reduced cell viability 


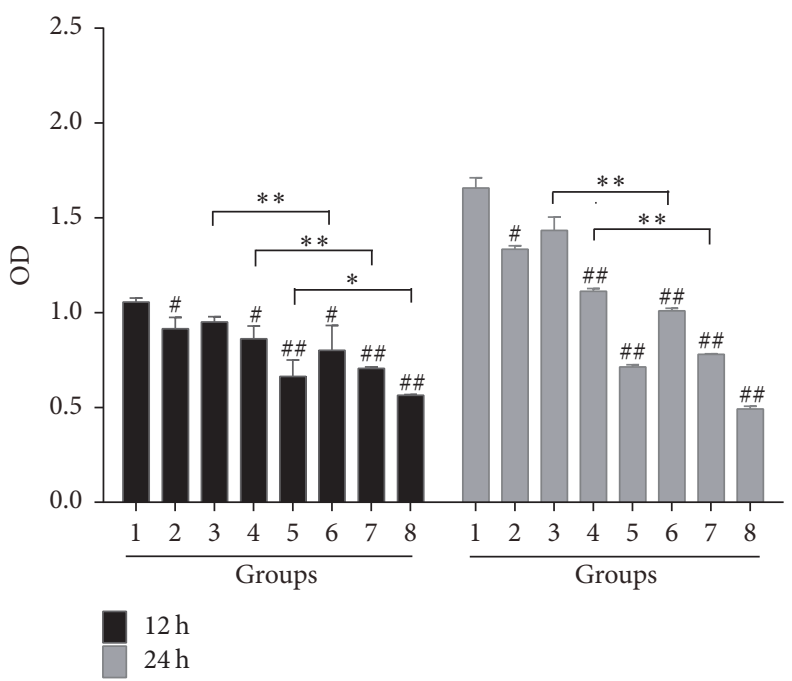

(a)

Characteristic morphological features of SMMC-7721 cells induced by DHZCP and DOX
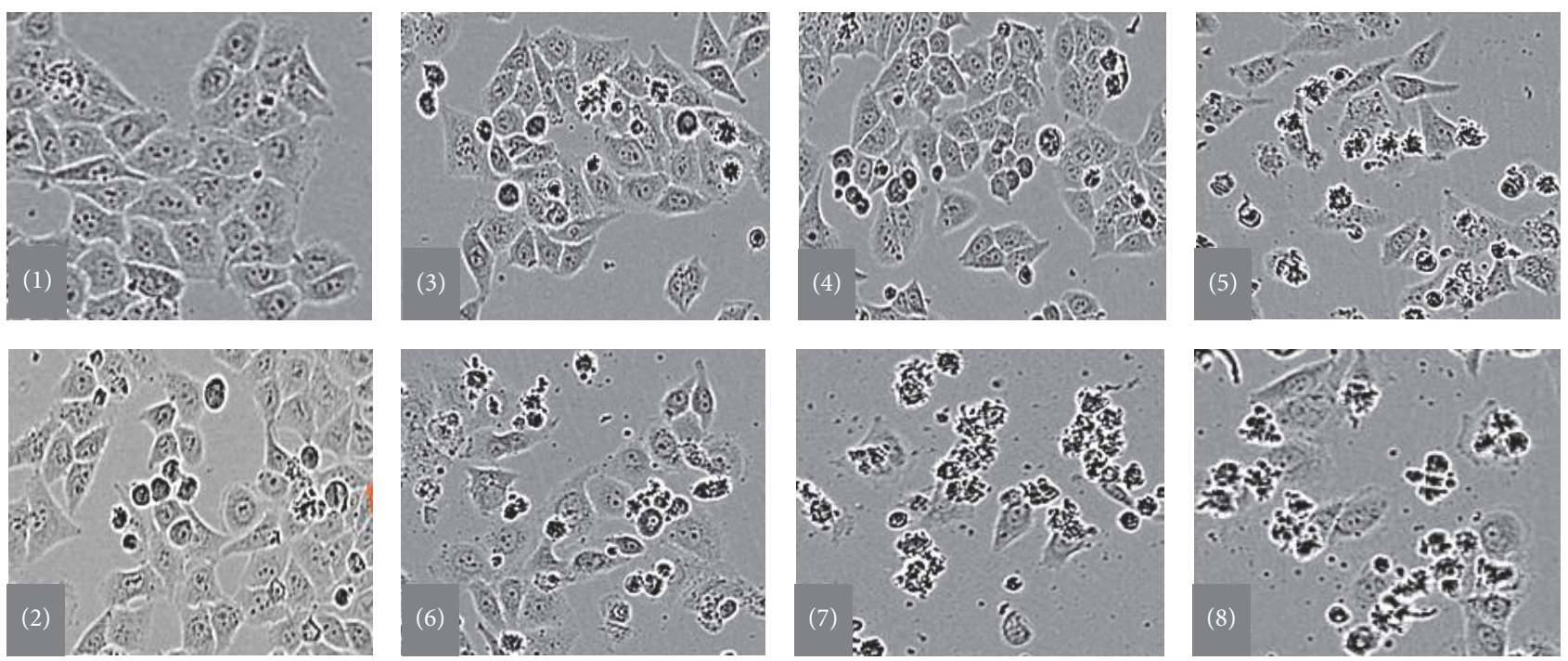

(b)

Figure 1: Effects of DHZCP-medicated serum combined with DOX on SMMC-7721 cell viability. (a) CCK-8 assay for evaluating cell viability. (b) Changes of cell configuration of SMMC-7721 treated with DHZCP-medicated serum and/or DOX. Groups: (1) control; (2) DHZCP-medicated serum; (3)-(5) DOX $(0.5,2$, and $8 \mu \mathrm{M})$; (6)-(8) combination of DHZCP-medicated serum with DOX $(0.5,2$, and $8 \mu \mathrm{M})$. Significance: ${ }^{\#} p<0.05,{ }^{\# \#} p<0.01$ in comparison with control; ${ }^{*} p<0.05,{ }^{* *} p<0.01$ compared with DOX group.

after $12 \mathrm{~h}$ incubation (Figure $1(\mathrm{a})$ ), and the inhibitory effects were more evident after $24 \mathrm{~h}$ treatment. However, DOX at $0.5 \mu \mathrm{M}$ had no noticeable effect on cell viability. When working in conjunction with DHZCP-medicated serum, the cell viability was more significantly reduced compared with treatment with DOX alone. Microscopic examinations indicated that the secretion of SMMC-7721 was notably increased and a large number of cells were dead (Figure 1(b)). It was noted that combination of DOX at $8 \mu \mathrm{M}$ with DHZCPmedicated serum showed no significant difference compared with each alone, which might be due to excessive cell death caused by DOX at high concentration.
3.2. Impacts of DHZCP-Medicated Serum Combined with DOX on SMMC-7721 Cell Apoptosis. Based on the detection of cell viability, the effects of DOX and DHZCPmedicated serum on SMMC-7721 cell apoptosis were further investigated. The results suggested that apoptosis of cells treated with 10\% DHZCP-medicated serum for $24 \mathrm{~h}$ was significantly increased (Figure 2(b)). There was no evident variation in apoptosis among treatments with DOX at $0.5 \mu \mathrm{M}$ alone. But treatment with DOX at $2 \mu \mathrm{M}$ for $12 \mathrm{~h}$ induced cell apoptosis and its combination with DHZCPmedicated serum lead to more noticeable apoptosis (Figure $2(\mathrm{~d})$ ), and only a few necrotic cells appeared. Moreover, 


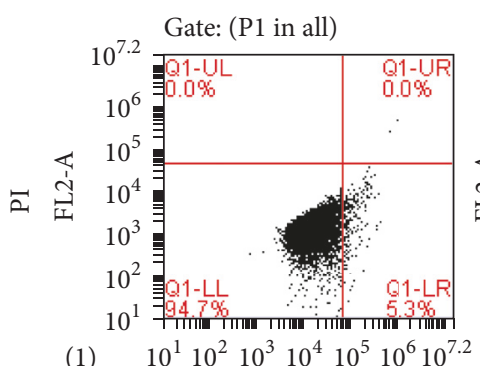

(1) $10^{1} 10^{2} 10^{3} 10^{4} 10^{5} 10^{6} 10^{7.2}$

FL1-A

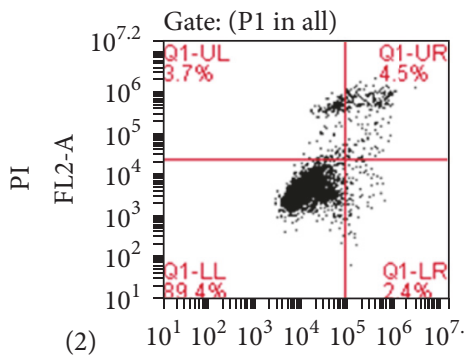

(2) $10^{1} 10^{2} 10^{3} 10^{4} 10^{5} 10^{6} 10^{7.2}$

FL1-A

Annexin V-FITC

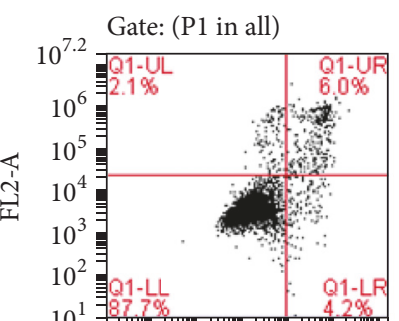

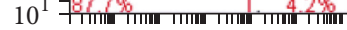

(3) $10^{1} 10^{2} 10^{3} 10^{4} 10^{5} 10^{6} 10^{7.2}$

FL1-A

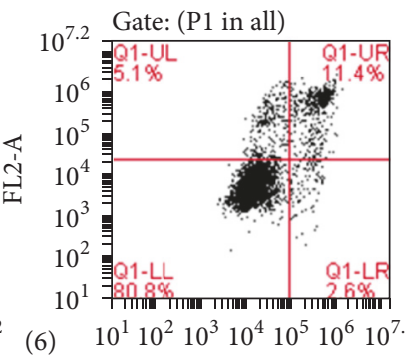

(6) $10^{1} 10^{2} 10^{3} 10^{4} 10^{5} 10^{6} 10^{7.2}$

FL1-A
Annexin V-FITC

(a)

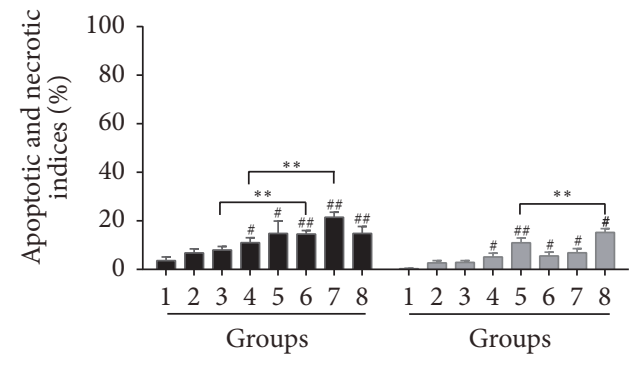

Apoptotic index

Necrotic index

(b)

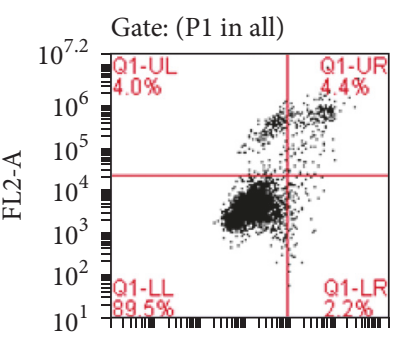

(4) $10^{1} 10^{2} 10^{3} 10^{4} 10^{5} 10^{6} 10^{7.2}$

FL1-A

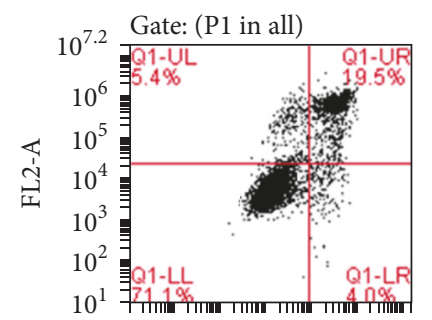

(7) $10^{1} 10^{2} 10^{3} 10^{4} 10^{5} 10^{6} 10^{7.2}$

FL1-A

Annexin V-FITC

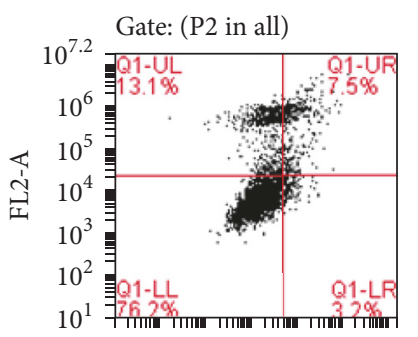

(5) $10^{1} 10^{2} 10^{3} 10^{4} 10^{5} 10^{6} 10^{7.2}$

FL1-A

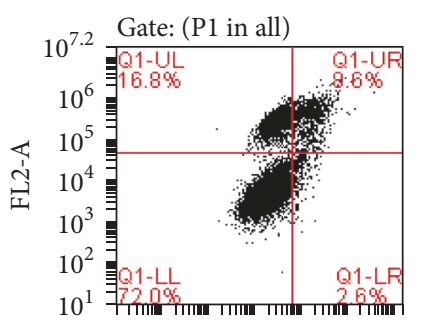

(8) $10^{1} 10^{2} 10^{3} 10^{4} 10^{5} 10^{6} 10^{7.2}$

FL1-A

Annexin V-FITC

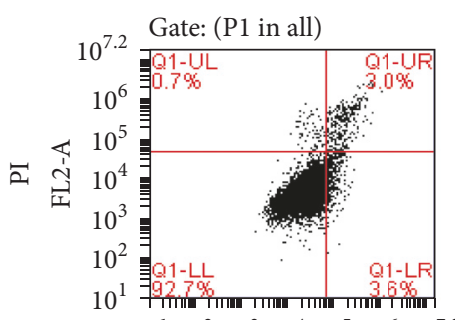

(1) $10^{1} 10^{2} 10^{3} 10^{4} 10^{5} 10^{6} 10^{7.2}$

$$
\text { FL1-A }
$$

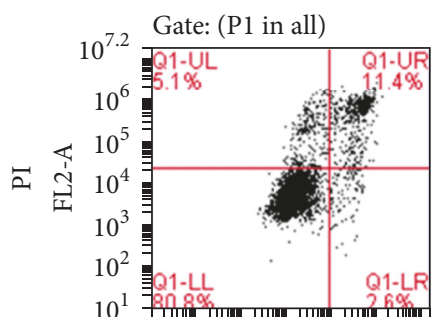

(2) $10^{1} 10^{2} 10^{3} 10^{4} 10^{5} 10^{6} 10^{7.2}$ FL1-A Annexin V-FITC

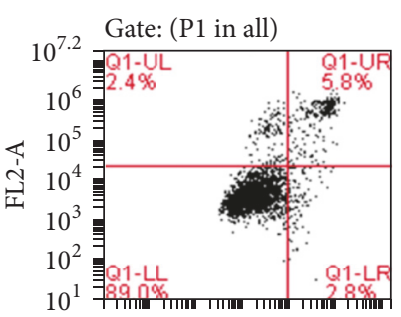

(3) $10^{1} 10^{2} 10^{3} 10^{4} 10^{5} 10^{6} 10^{7.2}$ FL1-A

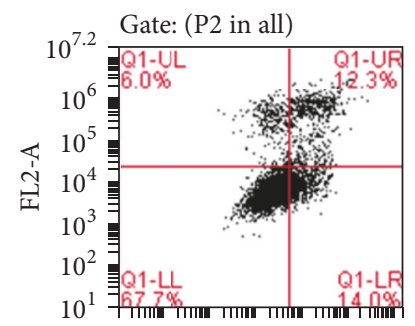

(6) $10^{1} 10^{2} 10^{3} 10^{4} 10^{5} 10^{6} 10^{7.2}$ FL1-A Annexin V-FITC

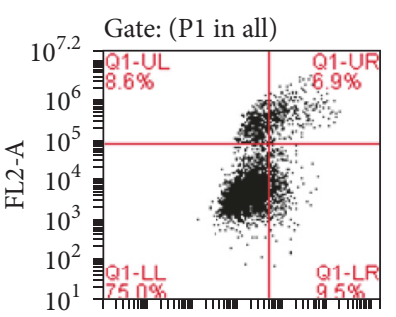

(4) $10^{1} 10^{2} 10^{3} 10^{4} 10^{5} 10^{6} 10^{7.2}$

FL1-A

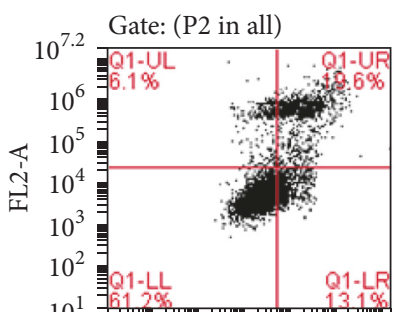

(7) $10^{1} 10^{2} 10^{3} 10^{4} 10^{5} 10^{6} 10^{7.2}$

FL1-A

Annexin V-FITC

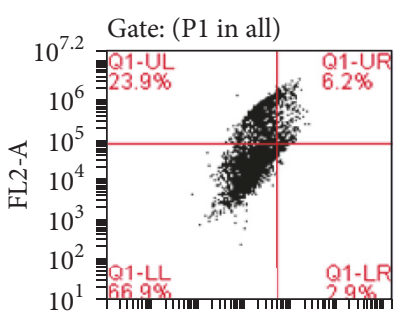

(5) $10^{1} 10^{2} 10^{3} 10^{4} 10^{5} 10^{6} 10^{7.2}$

FL1-A

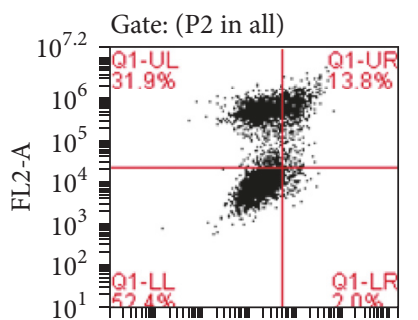

(8) $10^{1} 10^{2} 10^{3} 10^{4} 10^{5} 10^{6} 10^{7.2}$

FL1-A

Annexin V-FITC

(c)

FIgure 2: Continued. 


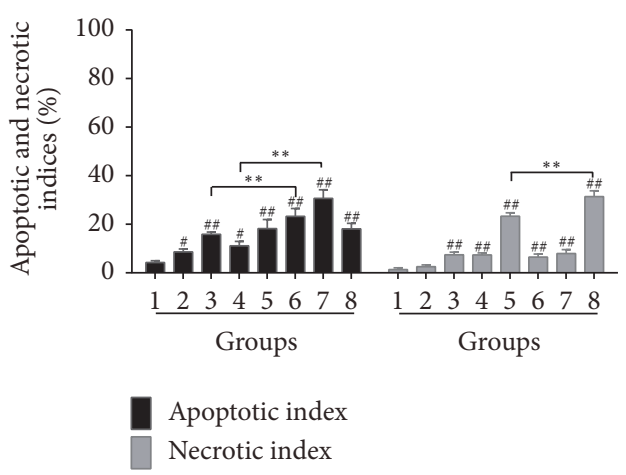

(d)

FIGURE 2: Impacts of DHZCP-medicated serum and/or DOX on SMMC-7721 cell apoptosis and necrosis. (a) and (c) Flow cytometric analyses. (b) and (d) Apoptotic and necrotic indices of SMMC-7721 dealt with DHZCP with or without DOX. Groups: (1) control; (2) DHZCP-medicated serum; (3)-(5) DOX $(0.5,2$, and $8 \mu \mathrm{M})$; (6)-(8) combination of DHZCP-medicated serum with DOX (0.5, 2, and $8 \mu \mathrm{M})$. Significance: ${ }^{\#} p<0.05,{ }^{\# \#} p<0.01$ versus control; ${ }^{* *} p<0.01$ compared with DOX group.

DOX at high dose $(8 \mu \mathrm{M})$ induced apoptosis of SMMC7721 cells accompanied by partial necrosis. When combined with DHZCP-medicated serum, the number of apoptotic cells (Figure 2(a)) and necrotic cells was raised evidently (Figure 2(c)). It was observed that the number of necrotic cells was increased concentration-dependently when treated with DHZCP-medicated serum combined with DOX, but the number of apoptotic cells remained unchanged after treatment for $24 \mathrm{~h}$.

\subsection{Role of DHZCP-Medicated Serum Combined with DOX} on Endogenous ATP Levels in SMMC-7721. Apoptosis and necrosis are two important forms in cell death. In response to drug stimulation, the choice of apoptosis or necrosis is determined by intracellular levels of ATP [7]. Therefore, we further investigated the effects of DHZCP-medicated serum and/or DOX on ATP levels in SMMC-7721. The outcome exhibited that the intracellular levels of ATP were significantly decreased in SMMC-7721 incubated with DHZCPmedicated serum for $12 \mathrm{~h}$ and $24 \mathrm{~h}$ (Figure 3 ). By contrast, the effects of DOX on ATP were much more complex. The levels of ATP in SMMC-7721 cells incubated with DOX at low concentration $(0.5 \mu \mathrm{M})$ for $12 \mathrm{~h}$ changed indistinctly while it elevated markedly after $24 \mathrm{~h}$ stimulation. The levels of ATP were increased distinctly under the stimulation of middle concentration of DOX $(2 \mu \mathrm{M})$ for $12 \mathrm{~h}$, and it augmented continuously for $24 \mathrm{~h}$. However, intracellular ATP levels dropped consistently after treatment with DOX at high concentration $(8 \mu \mathrm{M})$. These results revealed that DHZCPmedicated serum could reverse the increase of ATP with lower concentrations of DOX $(0.5$ and $2 \mu \mathrm{M})$ and further diminished the intracellular levels of ATP combined with a high concentration of DOX.

3.4. Functions of DHZCP-Medicated Serum Combined with DOX on Oxidative Phosphorylation Enzymes in SMMC-7721. Different from normal cells, aerobic fermentation exerts a prominent importance to the energy metabolism of HCC cells in addition to mitochondrial oxidative phosphorylation pathway. Therefore, effects of DHZCP-medicated serum

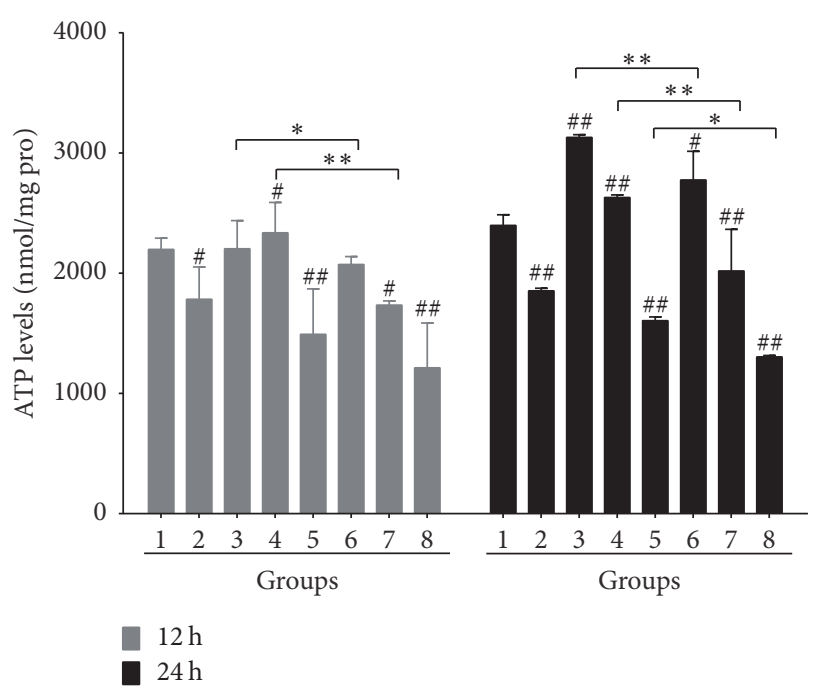

FIGURE 3: Role of DHZCP-medicated serum and/or DOX on intracellular ATP levels in SMMC-7721. Groups: (1) control; (2) DHZCP-medicated serum; (3)-(5) DOX (0.5, 2, and $8 \mu \mathrm{M})$; (6)-(8) combination of DHZCP-medicated serum with DOX $(0.5,2,8 \mu \mathrm{M})$. Significance: ${ }^{\#} p<0.05,{ }^{\# \#} p<0.01$ compared with control; ${ }^{*} p<$ $0.05,{ }^{* *} p<0.01$ compared with DOX group.

combined with DOX on the key enzymes involved in oxidative phosphorylation and glycolysis pathways in SMMC-7721 were further examined by Western blotting (Figure 4(a)). The results suggested that treatment with DHZCP-medicated serum for $24 \mathrm{~h}$ significantly reduced the expressions of a series of oxidative phosphorylation complexes including CII, CIII, and CV (Figure 4(b)) in SMMC-7721. But DHZCPmedicated serum had no significant effects on the key enzymes of the glycolytic pathway. DOX at different concentrations had different effects on these oxidative phosphorylation enzymes. DOX at lower concentrations (0.5 and $2 \mu \mathrm{M})$ can not only increase the expression of $\mathrm{HK}$ involved in glycolytic pathway (Figure 4(c)) but also increase the expressions of complexes of oxidative phosphorylation 


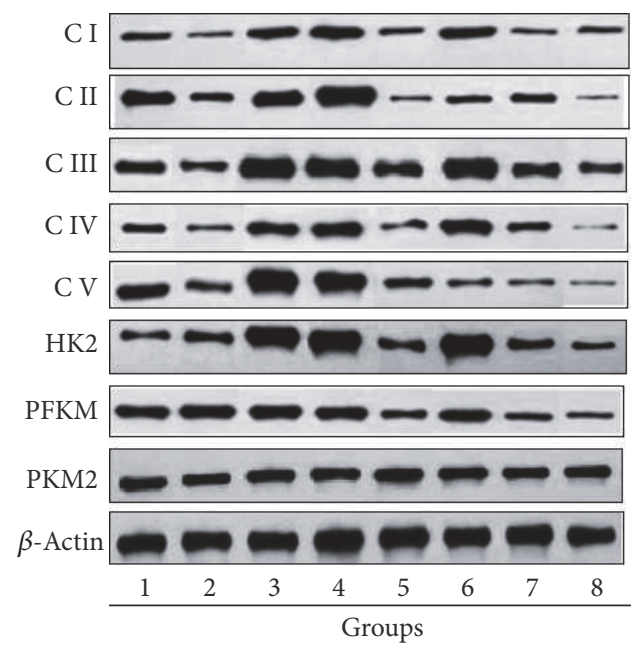

(a)

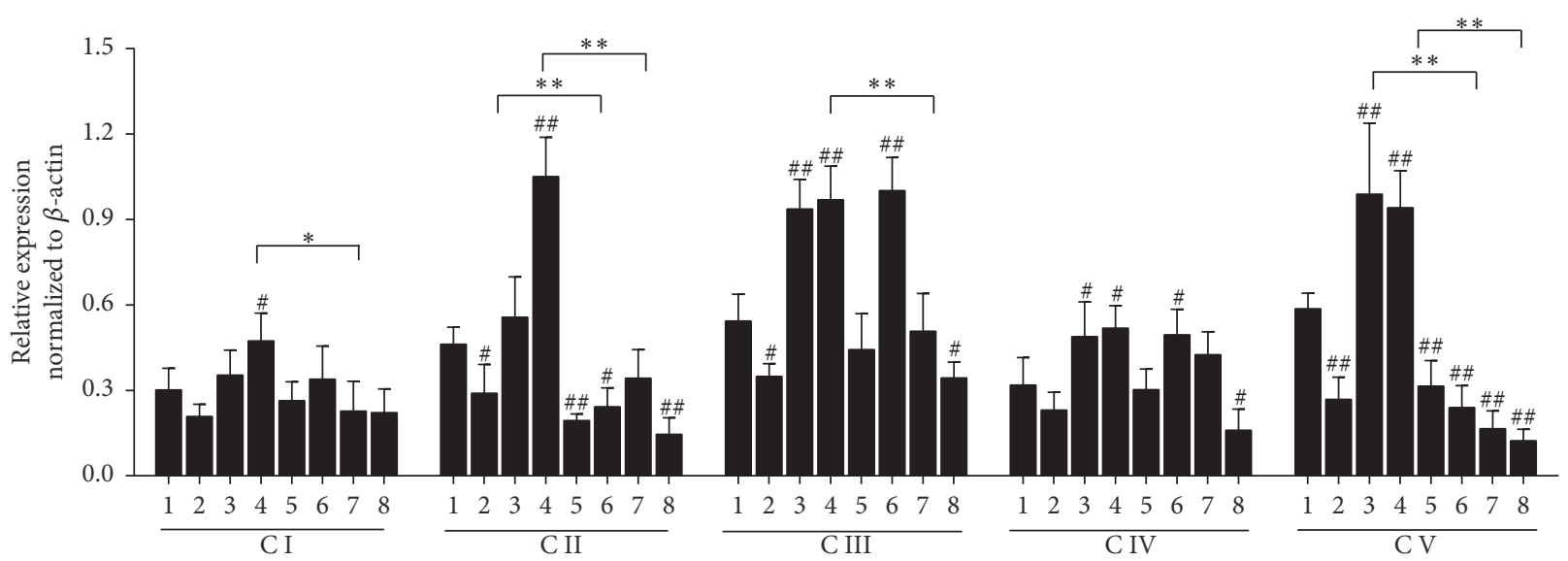

(b)

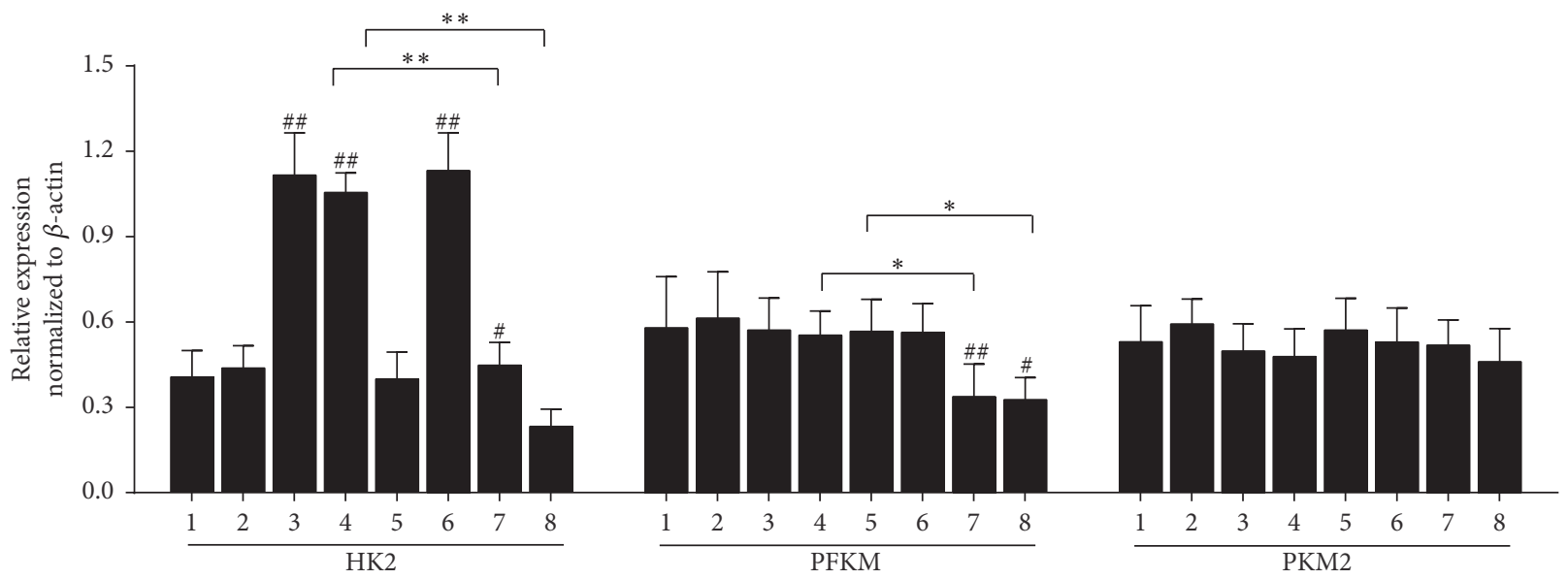

(c)

FIGURE 4: Effects of DHZCP-medicated serum with or without DOX on oxidative phosphorylation enzymes involved in oxidative phosphorylation and glycolysis in SMMC-7721. (a) Western blot analyses of expression of CI, CII, CIII, CIV, CV, HK2, PFKM, and PKM2. (b) and (c) Relative expression normalized to $\beta$-actin. Groups: (1) control; (2) DHZCP-medicated serum; (3)-(5) DOX (0.5, 2, and 8 $\mu \mathrm{M})$; (6)-(8) combination of DHZCP-medicated serum with $\operatorname{DOX}(0.5,2$, and $8 \mu \mathrm{M})$. Significance: ${ }^{\#} p<0.05$, \#\# $p<0.01$ in comparison with control; ${ }^{*} p<0.05,{ }^{* *} p<0.01$ compared with DOX group. 
pathway. In contrast, DOX at high concentration $(8 \mu \mathrm{M})$ decreased the expressions of a variety of oxidative phosphorylation enzymes, including $\mathrm{CII}$ and $\mathrm{CV}$. In addition, the combination of DHZCP-medicated serum and DOX at 0.5 or $2 \mu \mathrm{M}$ reversed the increased oxidative phosphorylation by DOX, and significant differences in CI, CII, CV, and CIII compared with DOX alone were observed. However, the combination of DHZCP-medicated serum and DOX at $8 \mu \mathrm{M}$ markedly decreased the expressions of key enzymes of oxidative phosphorylation. Simultaneously, the expressions of PFKM and HK in glycolytic pathway were also significantly decreased.

\section{Discussion}

DOX is a broad-spectrum antibiotic which is commonly applied in clinical treatments of tumors. It can be inserted into nuclear DNA and inhibit topoisomerase II, leading to the damage of DNA tertiary structure. Thus, it is especially useful for killing tumor cells at a rapid growth stage [8]. In this research, we found that DOX exerted its antitumor effects on SMMC-7721 by inducing cell death in a dosedependent way. Specifically, DOX can promote apoptosis at low or middle concentration and mainly induced necrosis at high concentration. DHZCP-medicated serum combined with DOX at low concentration could significantly increase the number of apoptotic cells and combined with DOX at high concentration it could markedly increase the number of necrotic cells, suggesting synergistic antihepatoma effects.

Necrosis and apoptosis are two important forms of cell death. Apoptosis is considered as an active process that consumes ATP, and necrosis is a passive process after ATP depletion $[9,10]$. However, it has also been accepted that necrosis and apoptosis often appeared in the same tissues [11]. And apoptosis could turn into necrosis at the condition of an acute decline of intracellular ATP level [12-15]. Our experiments found that effects of DOX on intracellular ATP levels were dose-dependent. The expressions of key enzymes involved in oxidative phosphorylation, glycolytic pathways, and ATP levels were apparently elevated in SMMC7721 treated with DOX at low or middle concentration and decreased in SMMC-7721 treated with DOX at high concentration dramatically. Was the variation of endogenous ATP levels the direct result of the cytotoxicity of DOX or the emergency response of cancer cells to drug toxicity?

As is known to all, almost all biological activities need to consume energy such as cell survival, proliferation, defense or adaptation, metabolism, signal transduction, and genetic expression. Liver cancer cells can quickly start stress response requiring ATP upon chemotherapy. For example, drug-efflux and antiapoptotic pathways were established or the structures of drug targets were changed. To a certain extent, these behaviors seriously reduced the therapeutic effects of chemotherapeutic drugs and led to MDR [16]. For example, after stimulation with chloroethyl nitrosourea, the mitochondrial energy metabolism of HepG2 cells could be rapidly activated and the cells were conferred a significant function of antiapoptosis [17]. Glucose deprivation in HepG2 cells culture medium also significantly activated the mitochondrial respiratory chain and increased the expression of ATP synthase $\beta$ subunit [18]. Recent studies showed that a newly developed anticancer drug Casiopeina could also inhibit the oxidative phosphorylation and glycolytic processes in hepatoma cells, and the anticancer effects were obviously better than those of glycolytic inhibitor 3-bromothiophene [19]. These results suggested that liver cancer cells of the mitochondrial pathway could be activated under stress conditions such as hypoxia and anticancer drug chemotherapy, because this activation might provide a great deal of ATP to launch a variety of defensive mechanisms to drugs (efflux pump, DNA damage repair, upregulation of survival-related genes, and antiapoptotic machinery). These factors are the important causes for MDR of chemotherapy and the failure of treatment of liver cancer. So far, we found that DOX prominently reduced ATP levels at high concentration presumably because of the activation of PARP (poly(ADP-ribose) polymerase) and the consumption of a large amount of nicotinamide adenine dinucleotide $\left(\mathrm{NAD}^{+}\right)$.

\section{Conclusion}

DHZCP from "Synopsis of Prescriptions of the Golden Chamber (Jin Kui Yao Lue)" written by Zhang was composed of twelve traditional Chinese medicinal herbs [20], and several kinds of herbs in DHZCP can inhibit hepatic mitochondrial energy metabolism, which is considered to be one of the common characteristics of TCM with cold property [21-24]. This study suggested that DHZCP could downregulate the expressions of critical enzymes of oxidative phosphorylation and reduce the intracellular ATP levels in hepatoma carcinoma SMMC-7721 cells. When combined with DNA-targeted DOX, DHZCP could reverse the elevation of intracellular ATP levels induced by DOX. This property of DHZCP could not only enhance the therapeutic effects of chemotherapeutic drugs but also reduce the doses of chemotherapeutic drugs and the incidences of adverse reactions.

\section{Conflicts of Interest}

All authors declare no conflicts of interest.

\section{Authors' Contributions}

Li Wu, Jiayu Zhao, and Hao Cai contributed equally to this work.

\section{Acknowledgments}

This work was supported by the National Natural Science Foundation of China (81503308, 81673600, and 81473147), the Project of Administration of Traditional Chinese Medicine of Jiangsu Province (YB2015007), the Top-Notch Academic Programs Project of Jiangsu Higher Education Institutions (PPZY2015A070), the Postgraduate Research Innovation Project of Jiangsu Higher Education Institutions (KYLX16_1160), the Project Funded by the Priority Academic 
Program Development of Jiangsu Higher Education Institutions (PAPD), and Top-Notch Academic Programs Project of Jiangsu Higher Education Institutions (TAPP).

\section{References}

[1] G. Ranieri, I. Marech, V. Lorusso et al., "Molecular targeting agents associated with transarterial chemoembolization or radiofrequency ablation in hepatocarcinoma treatment," World Journal of Gastroenterology, vol. 20, no. 2, pp. 486-497, 2014.

[2] M. Saraswathy and S. Gong, "Different strategies to overcome multidrug resistance in cancer," Biotechnology Advances, vol. 31, no. 8, pp. 1397-1407, 2013.

[3] R. Silva, V. Vilas-Boas, H. Carmo et al., "Modulation of Pglycoprotein efflux pump: Induction and activation as a therapeutic strategy," Pharmacology and Therapeutics, vol. 149, pp. 1-123, 2015.

[4] A. Palmeira, E. Sousa, M. H. Vasconcelos, and M. M. Pinto, "Three decades of P-gp Inhibitors: Skimming through several generations and scaffolds," Current Medicinal Chemistry, vol. 19, no. 13, pp. 1946-2025, 2012.

[5] J. Wu and H. D. Shen, "Pill of Dahuang Zhechong Wan with chemotherapy in the treatment of primary carcinoma of liver at middle-advanced stage," Western Journal of Traditional Chinese Medicine, vol. 24, no. 10, pp. 54-55, 2011.

[6] L. Zhou, "Clinical observation of Dahuang Zhechong Pill combined with gemcitabine and oxaliplatin in the treatment of primary carcinoma of liver," Journal of New Chinese Medicine, vol. 46, no. 2, pp. 161-163, 2014.

[7] C. Wang, H. Chen, M. Zhang, J. Zhang, X. Wei, and W. Ying, "Malate-aspartate shuttle inhibitor aminooxyacetic acid leads to decreased intracellular ATP levels and altered cell cycle of C6 glioma cells by inhibiting glycolysis," Cancer Letters, vol. 378, no. 1, pp. 1-7, 2016.

[8] Q. J. Liu, Y. X. Tian, and Y. N. Xiao, "The effect of adriamycin local chemotherapy for cancer," Journal of Oncology, vol. 10, no. 1, pp. 45-47, 2004.

[9] M. Takeda, I. Shirato, M. Kobayashi, and H. Endou, "Hydrogen peroxide induces necrosis, apoptosis, oncosis and apoptotic oncosis of mouse terminal proximal straight tubule cells," Nephron, vol. 81, no. 2, pp. 234-238, 1999.

[10] S. H. Chang, P. C. Phelps, I. K. Berezesky, J. Ebersberger M.L., and B. F. Trump, "Studies on the mechanisms and kinetics of apoptosis induced by microinjection of cytochrome $\mathrm{c}$ in rat kidney tubule epithelial cells (NRK-52E)," American Journal of Pathology, vol. 156, no. 2, pp. 637-649, 2000.

[11] C. J. Zeiss, "The apoptosis-necrosis continuum: Insights from genetically altered mice," Veterinary Pathology, vol. 40, no. 5, pp. 481-495, 2003.

[12] M. Leist, B. Single, A. F. Castoldi, S. Kühnle, and P. Nicotera, "Intracellular adenosine triphosphate (ATP) concentration: a switch in the decision between apoptosis and necrosis," The Journal of Experimental Medicine, vol. 185, no. 8, pp. 1481-1486, 1997.

[13] G. Denecker, D. Vercammen, W. Declercq, and P. Vandenabeele, "Apoptotic and necrotic cell death induced by death domain receptors," Cellular and Molecular Life Sciences, vol. 58, no. 3, pp. 356-370, 2001.

[14] N. I. Abdel-Hamid, M. F. El-Azab, and Y. M. Moustafa, "Macrolide antibiotics differentially influence human HepG2 cytotoxicity and modulate intrinsic/extrinsic apoptotic pathways in rat hepatocellular carcinoma model," NaunynSchmiedeberg's Archives of Pharmacology, vol. 390, no. 4, pp. 379-395, 2017.

[15] W. Fiers, R. Beyaert, W. Declercq, and P. Vandenabeele, "More than one way to die: apoptosis, necrosis and reactive oxygen damage," Oncogene, vol. 18, no. 54, pp. 7719-7730, 1999.

[16] R. Moreno-Sánchez, S. Rodríguez-Enríquez, E. Saavedra, A. Marín-Hernández, and J. C. Gallardo-Pérez, "The bioenergetics of cancer: Is glycolysis the main ATP supplier in all tumor cells?" BioFactors, vol. 35, no. 2, pp. 209-225, 2009.

[17] D. Loiseau, D. Morvan, A. Chevrollier et al., "Mitochondrial bioenergetic background confers a survival advantage to HepG2 cells in response to chemotherapy," Molecular Carcinogenesis, vol. 48, no. 8, pp. 733-741, 2009.

[18] R. Domenis, E. Bisetto, D. Rossi, M. Comelli, and I. Mavelli, "Glucose-modulated mitochondria adaptation in tumor cells: A focus on ATP synthase and Inhibitor Factor 1," International Journal of Molecular Sciences, vol. 13, no. 2, pp. 1933-1950, 2012.

[19] A. Marín-Hernández, J. C. Gallardo-Pérez, S. Y. López-Ramírez et al., "Casiopeina II-gly and bromo-pyruvate inhibition of tumor hexokinase, glycolysis, and oxidative phosphorylation," Archives of Toxicology, vol. 86, no. 5, pp. 753-766, 2012.

[20] Z. J. Zhang, Synopsis of prescriptions of the Golden Chamber (Jin Kui Yao Lue), The Eastern Han Dynasty, pp. 57.

[21] Y. Y. Wang, X. Sun, X. L. Pei, Q. H. Wang, B. Y. Yang, and H. X. Kuang, "Advances in researches on relations between cold and hot properties of TCM and mitochondrial energy metabolism," Information on Traditional Chinese Medicine, vol. 30, no. 4, pp. 48-50, 2013.

[22] L. P. Huang, S. H. Peng, X. F. Meng et al., "Study of hepatic energy metabolism on rats by six cold property traditional Chinese medicines," China Journal of Chinese Materia Medica, vol. 34, no. 24, pp. 3255-3258, 2009.

[23] Q. C. Ma, H. Y. Yu, J. Zhao et al., "Effects of monkshood, dry ginger, Chinese goldthread and rhubarb on energy metabolism of rats," Journal of Shandong University of TCM, vol. 34, no. 5, pp. 379-380, 2010.

[24] Y. Li, D.-Z. Lu, L.-H. Tang, X.-D. Wo, and Z. Yang, "Effect of cold and cool herbs on liver mitochondria proteome of rats with heat symptom," China Journal of Chinese Materia Medica, vol. 38, no. 24, pp. 4144-4147, 2013. 


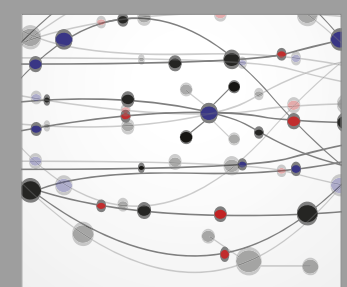

The Scientific World Journal
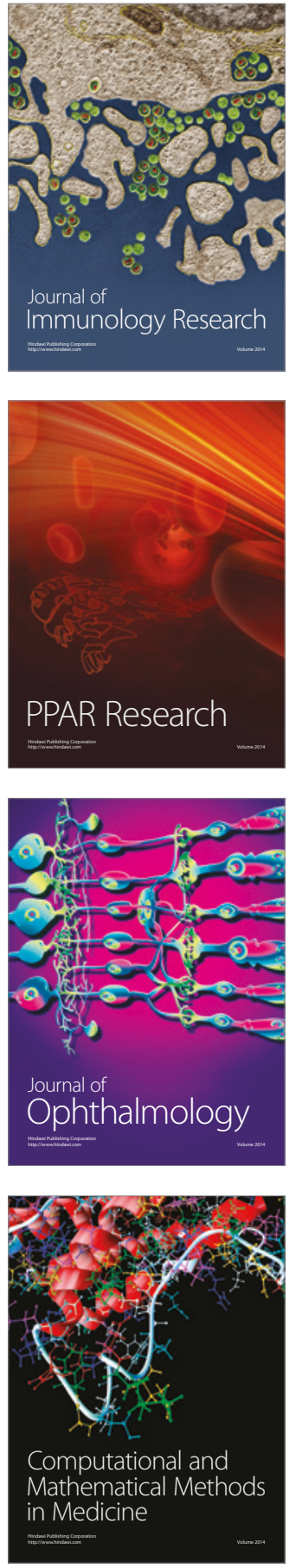

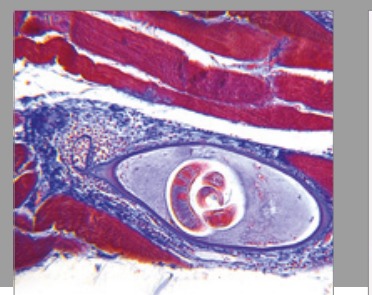

Gastroenterology Research and Practice
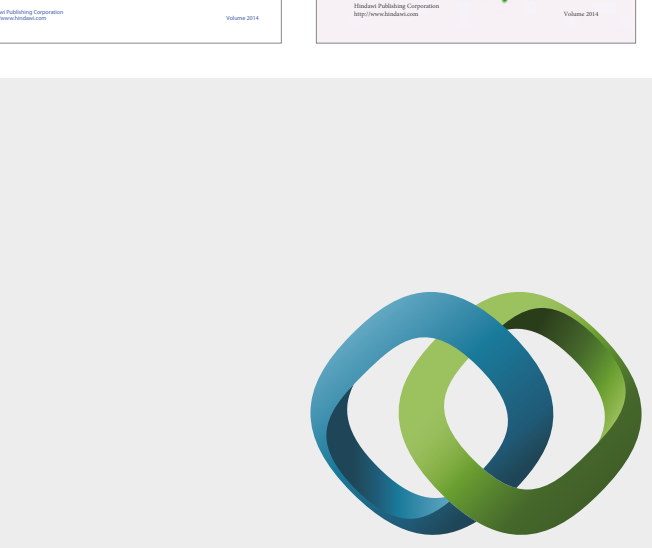

\section{Hindawi}

Submit your manuscripts at

https://www.hindawi.com
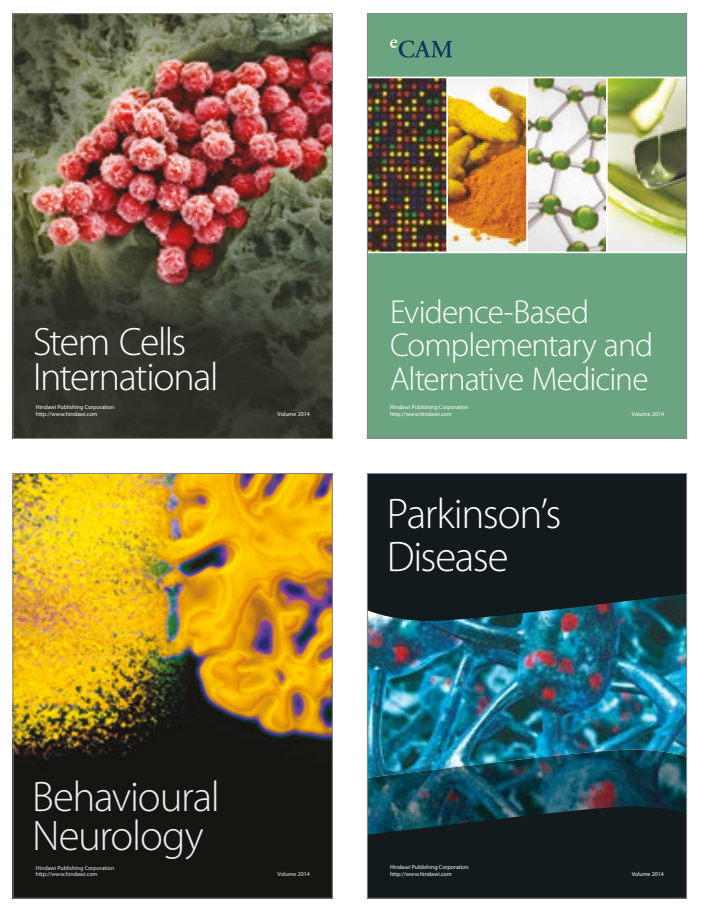
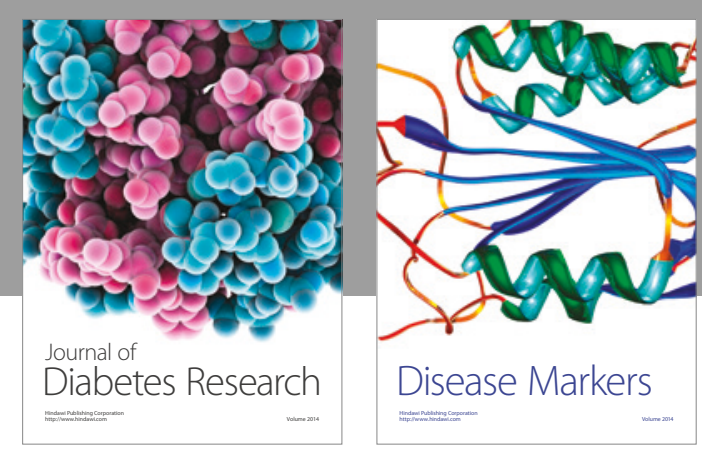

Disease Markers
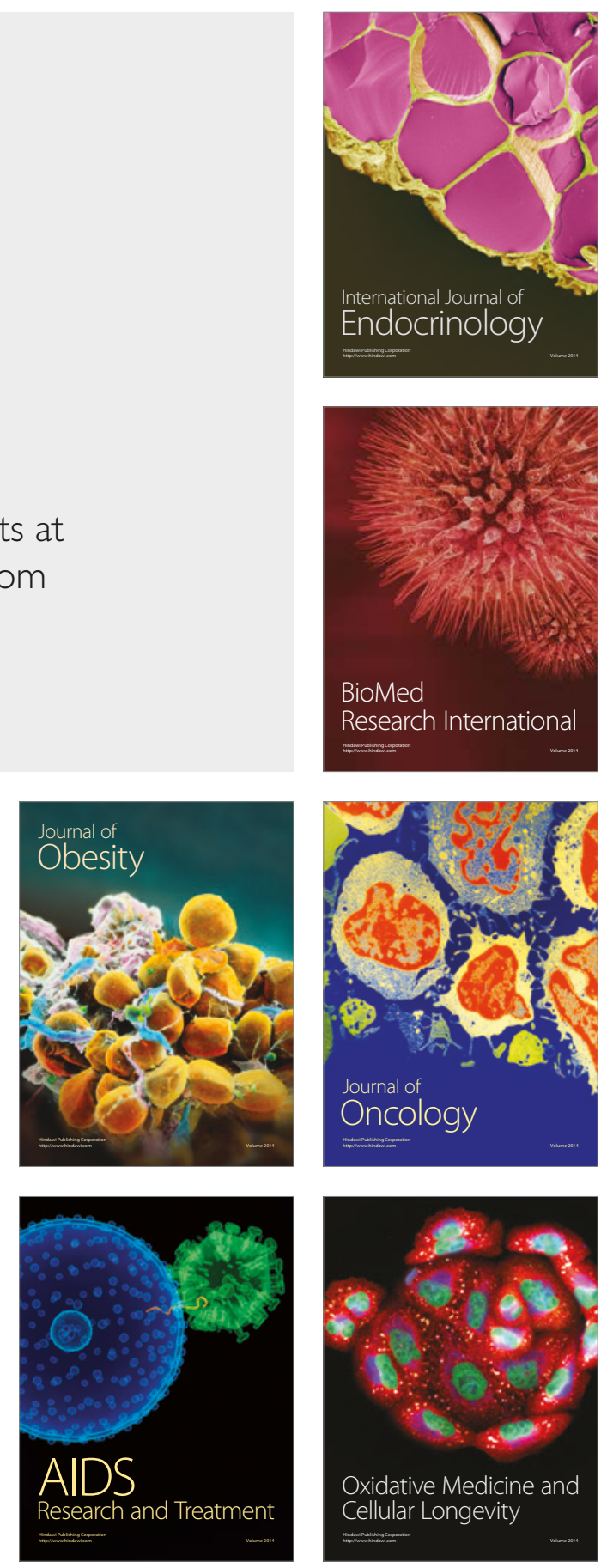